\title{
Abstract: Automated Segmentation of Bones for the Age Assessment in 3D MR Images using Convolutional Neural Networks
}

\author{
Markus Auf-der-Mauer ${ }^{1}$, Paul-Louis Pröve ${ }^{1}$, Eilin Jopp ${ }^{2}$, Jochen Herrmann ${ }^{3}$, \\ Michael Groth ${ }^{3}$, Michael M. Morlock ${ }^{4}$, Ben Stanczus ${ }^{1}$, Dennis Säring ${ }^{1}$ \\ ${ }^{1}$ Medical and Industrial Image Processing, University of Applied Sciences of Wedel \\ ${ }^{2}$ Department of Legal Medicine, University Medical Center Hamburg-Eppendorf \\ ${ }^{3}$ Pediatric Radiology Department, University Medical Center Hamburg-Eppendorf \\ ${ }^{4}$ Institute of Biomechanics, Hamburg University of Technology
}

adm@fh-wedel.de

The age assessment is a complicated procedure used to determine the chronological age of an individual who lacks legal documentation. Actual studies show that the ossification degree of the growth plates in the knee joint represents a suitable indicator for the majority age. To verify this hypothesis a high number of datasets have to be analysed. Therefore, approaches which enable the detection of the bone structures are necessary. In this work a fully automatic knee segmentation in 3D MR images using convolutional neural networks is presented. A total of 76 datasets were available and divided into a training set (74\%), a validation set (13\%) and a test set (13\%). Multiple preprocessing steps were applied to correct the images and to reduce their size. Image Augmentation was employed to virtually increase the data set size during training. The proposed architecture for the segmentation task resembles the encoder-decoder model type used for the U-Net. The trained network achieves a DSC score of $98 \%$ compared to the manual segmentations and an IoU of $96 \%$, outperforming the results by Dam et al.[1]. The precision and recall of the model are balanced and the error has a small value of $1.2 \%$. No overfitting was observed during training. As a proof of concept, the segmentations were used for the age estimation of a number of subjects. First results show the potential of this approach attaining a mean difference of $0.48 \pm 0.32$ years, which improve results from Stern et al. of 0.85 \pm 0.58 years [2]. In order to fully exploit the potential of neural networks and to supply a more precise and reliable age prediction, the approach has to be tested on a larger data collective. The proposed segmentation can lead to an improvement of age estimation methods.

\section{References}

1. Dam EB, Lillholm M, Marques J, et al. Automatic segmentation of high- and lowfield knee MRIs using knee image quantification with data from the osteoarthritis initiative. J Med Imaging. 2015;2(2):024001.

2. Stern D, Ebner T, Bischof H, et al. Fully automatic bone age estimation from left hand MR images. Proc MICCAI. 2014;17:220-227. 